\title{
Chronic Pain and the Emotional Brain: Specific Brain Activity Associated with Spontaneous Fluctuations of Intensity of Chronic Back Pain
}

\author{
Marwan N. Baliki, ${ }^{1}$ Dante R. Chialvo, ${ }^{1}$ Paul Y. Geha, ${ }^{1}$ Robert M. Levy, ${ }^{2}$ R. Norman Harden, ${ }^{5}$ Todd B. Parrish, ${ }^{3}$ and \\ A. Vania Apkarian ${ }^{1,4}$ \\ ${ }^{1}$ Departments of Physiology, ${ }^{2}$ Neurosurgery, ${ }^{3}$ Radiology, and ${ }^{4}$ Anesthesia, and ${ }^{5}$ Rehabilitation Institute of Chicago, Lurie Cancer Center, Northwestern \\ University, Feinberg School of Medicine, Chicago, Illinois 60611
}

\begin{abstract}
Living with unrelenting pain (chronic pain) is maladaptive and is thought to be associated with physiological and psychological modifications, yet there is a lack of knowledge regarding brain elements involved in such conditions. Here, we identify brain regions involved in spontaneous pain of chronic back pain (CBP) in two separate groups of patients ( $n=13$ and $n=11$ ), and contrast brain activity between spontaneous pain and thermal pain (CBP and healthy subjects, $n=11$ each). Continuous ratings of fluctuations of spontaneous pain during functional magnetic resonance imaging were separated into two components: high sustained pain and increasing pain. Sustained high pain of CBP resulted in increased activity in the medial prefrontal cortex (mPFC; including rostral anterior cingulate). This mPFC activity was strongly related to intensity of CBP, and the region is known to be involved in negative emotions, response conflict, and detection of unfavorable outcomes, especially in relation to the self. In contrast, the increasing phase of CBP transiently activated brain regions commonly observed for acute pain, best exemplified by the insula, which tightly reflected duration of $\mathrm{CBP}$. When spontaneous pain of CBP was contrasted to thermal stimulation, we observe a double-dissociation between $\mathrm{mPFC}$ and insula with the former correlating only to intensity of spontaneous pain and the latter correlating only to pain intensity for thermal stimulation. These findings suggest that subjective spontaneous pain of $\mathrm{CBP}$ involves specific spatiotemporal neuronal mechanisms, distinct from those observed for acute experimental pain, implicating a salient role for emotional brain concerning the self.
\end{abstract}

Key words: chronic pain; prefrontal cortex; thermal pain; emotion; BOLD; atrophy

\section{Introduction}

Pain is associated with negative emotions and is always highly salient, enabling the organism to either escape or protect the injured body part and thus enhance survival. However, when pain becomes chronic (Merskey and Bogduk, 1994), that is when subjects live with unrelenting pain over many years, it becomes maladaptive and modifies one's outlook to everyday experience and to future expectations, by changing physiological and psychological processes underlying pain perception and pain-related behavior. Such notions have been prevalent in pain research for a long time, and stem mainly from clinical observations (Melzack and Casey, 1968; Price, 2000; Dworkin, 2002). Here, we seek to identify brain elements that may be involved in these processes, specifically in chronic back pain (CBP).

Chronic pain is often associated with spontaneous pain (pain in the absence of external stimuli), as well as changes in sensitivity

Received June 16, 2005; revised 0ct. 13, 2006; accepted 0ct. 16, 2006.

This work was supported by National Institutes of Health-National Institute of Neurological Diseases Grant R01 NS 35115. We thank all patients and volunteers for their participation in this study. We also thank S. A. Khan, M. M. Mesulam, J. Gottfried, and D. Gitelman for reading previous versions of this manuscript.

Correspondence should be addressed to A. Vania Apkarian, Department of Physiology, Northwestern University Medical School, 5-120 Ward Building, 303 East Chicago Avenue, Chicago, IL 60611. E-mail: a-apkarian@northwestern.edu. DOI:10.1523/JNEUROSCI.3576-06.2006

Copyright $\odot 2006$ Society for Neuroscience $\quad 0270-6474 / 06 / 2612165-09 \$ 15.00 / 0$ to various somatosensory stimuli (Tasker et al., 1991; Clauw et al., 1999; Sindrup et al., 1999; Birklein et al., 2000; Dworkin, 2002). Approximately $10 \%$ of adults have severe chronic pain (Harstall and Ospina, 2003) and CBP is the largest contributor to this population (Atkinson, 2004). Such conditions diminish the quality of life, and increase anxiety and depression (Dworkin, 2002; Sah et al., 2003). Moreover, CBP is now associated with cognitive (Apkarian et al., 2004a), brain chemical (Grachev et al., 2000), and morphologic abnormalities (Apkarian et al., 2004b). Neuroimaging studies can provide complimentary evidence by demonstrating brain circuitry involved in the condition. Twobrain imaging studies have explored brain activity in CBP to thermal or mechanical painful stimuli applied to the hand, a site remote from the body area in pain (Derbyshire et al., 2002; Giesecke et al., 2004). Both studies indicate some differences in stimulus-evoked responses to these acute painful stimuli in CBP as compared with normal subjects. Neither study directly addresses brain activity related to spontaneous pain of CBP. Spontaneous pain is a main reason for subjects seeking medical care and is the primary complaint of CBP patients (Clauw et al., 1999; Dworkin, 2002). We have shown that intensity of chronic pain fluctuates spontaneously, and CBP patients instructed to indicate their pain intensity on a continuous scale exhibit fluctuations of 
spontaneous pain with unique properties (Foss et al., 2006). Here, we use these fluctuations of spontaneous pain, performed during functional magnetic resonance imaging (fMRI) scans, to study its underlying brain circuitry and contrast it to brain activity for acute thermal pain. We hypothesize that brain regions involved in spontaneous pain would reveal the cortical adaptive processes associated with chronic pain. Based on our previous observations, we also hypothesize that CBP should be associated with increased prefrontal cortical activity. The present study confirms this, and unravels its details. Additionally, we sought to identify markers that can link the brain activity with clinical characteristics of CBP, to establish at least correlative links between the condition and brain states, and examined the impact of brain morphological changes in CBP on brain activity, searching for a link between cortical reorganization and back pain.

\section{Materials and Methods}

\section{Subjects}

A total of $24 \mathrm{CBP}$ patients and 11 healthy subjects participated in this study. All patients were clinically diagnosed with CBP. Participants were divided into three separate groups: group 1 consisted of 13 CBP patients scanned while rating spontaneous pain, and only participated in the first study. Group 2 consisted of 11 CBP patients who were scanned while rating their spontaneous pain and also for rating pain in response to thermal stimulus applied to their back. Group 3 consisted of 11 normal subjects matched for sex and age with patients of group 2 and rated their pain in response to an external thermal stimulus applied to their back. Groups 2 and 3 participated in study 2. Clinical and demographic data, as well as pain-related parameters for all participants are presented in supplemental Table 1, available at www.jneurosci.org as supplemental material.

\section{Pain ratings and experimental conditions}

Subjects used a finger-spanning device to continuously rate and log their subjective perception of pain during fMRI data collection (Apkarian et al., 2001). All participants underwent an initial training phase before scanning in which they learned to use a finger-span device to rate the size of a bar varying in time, at a rate approximating variability of spontaneous CBP. During brain imaging sessions, the finger-span device was synchronized and time locked with fMRI image acquisition and connected to a computer providing visual feedback. This device was also used in performing visual control scans.

Condition 1: rating of the spontaneous fluctuations of back pain. The CBP patients (group1 and 2) simply rated spontaneous fluctuations in the intensity of their ongoing pain in the absence of any external stimulus on a scale from 0 to 10 , with zero representing no pain and 10 representing maximum imaginable pain. An example of this rating is shown in Figure $1 \mathrm{~A}$. Subjects were provided with visual feedback of their rating by observing a scaled bar reflecting their current rating on a computer monitor.

Condition 2: rating pain in response to thermal stimulation. Subjects in groups 2 and 3 were scanned during acute thermal stimulation where they rated the stimulus pain with the finger-span device. During a given functional imaging session, eight noxious thermal stimuli ranging in duration from 10 to $30 \mathrm{~s}$ were applied to the lower back of either CBP patients (group 2) or healthy subjects (group 3). Similar to condition 1, subjects were instructed to rate their pain on a scale of $0-10$ and were provided with a visual feedback of their rating. A purpose built, fMRIcompatible thermal stimulator delivered fast ramping $\left(20^{\circ} \mathrm{C}\right.$ per second $)$ painful thermal stimuli (baseline $38^{\circ} \mathrm{C}$, peak temperatures 48 and $50^{\circ} \mathrm{C}$ ) via a contact probe $(1 \times 1.5 \mathrm{~cm}$ peltier $)$. Durations and intensities of thermal stimuli as well as interstimulus intervals were presented in a pseudorandom manner. They were designed to approximate the pattern of variability, and range of intensities, observed for spontaneous CBP. An example of a stimulus run and the corresponding group-average pain ratings for CBP and healthy controls are shown in Figure $5 \mathrm{~A}$. The inter- stimulus intervals ranged from 30 to $60 \mathrm{~s}$ (average of $55 \mathrm{~s}$ ). This variation in interval was adopted to decrease the regularity of stimulus presentation and thus reduce volunteers' ability to predict the arrival of the next stimulus. In addition, the relatively long duration interstimulus interval reduced sensitization.

Condition 3: visual control (all groups). Subjects were instructed to follow as closely as possible fluctuations of a bar projected on a screen in time. Unbeknown to the subjects, the variability of the bar was derived from previous functional scans where the participants were rating either their spontaneous pain or their thermal stimulus pain. This visual tracking provides an adequate visual-motor-cognitive control because it is similar to the pain rating finger-span task, with the important difference being that now the finger movement (i.e., variations in magnitude and its assessment) is correlated with a visual input rather than pain.

\section{Functional magnetic resonance data and analysis}

Study 1. Only CBP patients from group 1 participated in this study. fMRI data were acquired with a $1.5 \mathrm{~T}$ Siemens (Erlangen, Germany) (Vision) whole-body scanner with echo-planar imaging (EPI) capability using the standard radio-frequency head coil. Multislice $\mathrm{T} 2^{*}$-weighted echoplanar images were obtained with the following parameters: repetition time (TR), $3.5 \mathrm{~s}$; echo time (TE), $70 \mathrm{~ms}$; flip angle, $90^{\circ}$; slice thickness, 3 $\mathrm{mm}$; in-plane resolution, $3.475 \times 3.475 \mathrm{~mm}^{2}$. The 36 slices covered the whole brain from the cerebellum through to the vertex. A No-Flow T1weighted anatomical MRI image was also acquired for each subject using the following parameters: TR, $22 \mathrm{~ms}$; TE, $5.6 \mathrm{~ms}$; flip angle, $20^{\circ}$; matrix $256 \times 256$; and a field of view (FOV) of $240 \mathrm{~mm}$, with $160 \mathrm{~mm}$ coverage in the slice direction. Anatomic images were used to register functional images in standard space.

Image analysis to reveal significant brain activity based on changes in blood oxygen level-dependent (BOLD) signal was performed on each subject's data using Functional Magnetic Resonance Imaging of the Brain (FMRIB) Expert Analysis Tool [FEAT (Jezzard et al., 2001); www. fmrib.ox.ac.uk/fsl]. The preprocessing of each subject's time-series of fMRI volumes encompassed slice time correction, motion correction using MCFLIRT (motion correction using FMRIB's Linear Image Registration Tool), spatial smoothing using a Gaussian kernel of full-widthhalf-maximum $5 \mathrm{~mm}$, and nonlinear high-pass temporal filtering and subtraction of the mean of each voxel time course from that time course (i.e., intensity normalization). The fMRI signal was then linearly modeled on a voxel by voxel basis using FMRIB's Improved Linear Model (FILM) with local autocorrelation correction (Woolrich et al., 2001, 2004).

Two components were derived from the rating of spontaneous fluctuations in the intensity of ongoing CBP to search for brain activity. (1) To identify brain activity for time periods when the ongoing pain is high versus low, pain ratings were binarized relative to mean pain. (2) To identify brain activity for time periods when subjective pain is increasing: time epochs of ratings when positive rate of change of rating was sustained for a minimum time period ( $7 \mathrm{~s} ; 2 \mathrm{TRs})$ relative to all other time periods were binarized. An example of these components is presented in Figure 1. The visual vector was also derived in a similar manner (i.e., was binarized relative to the mean). In addition to the visual control, a surrogate control was generated for group 1 by inverting in time the recorded pain rating. This procedure preserves all statistical properties of the original ratings, but scrambles the relationship between the ratings and the actual pain fluctuations, thus controlling for nonspecific activations. Each component (pain and visual) was considered to generate a hemodynamic response described by the convolution of the corresponding vector with a generalized hemodynamic response function (gamma function: lag, $6 \mathrm{~s} ; \mathrm{SD}, 3 \mathrm{~s}$ ). Head motion vector (derived from motion correction) was used at this level as a covariate of no interest to further remove any residual variance caused by head motion. The significance of the model fit to each voxel time series was calculated, yielding statistical parametric maps for each subject and condition. Average group activity maps were generated by subtracting the visual and surrogate activity maps from the pain activity map using FEAT in a second level random, and fixed, effects group analysis after the coregistration of individual scans to standard space [152 subject average Montreal Neurological 

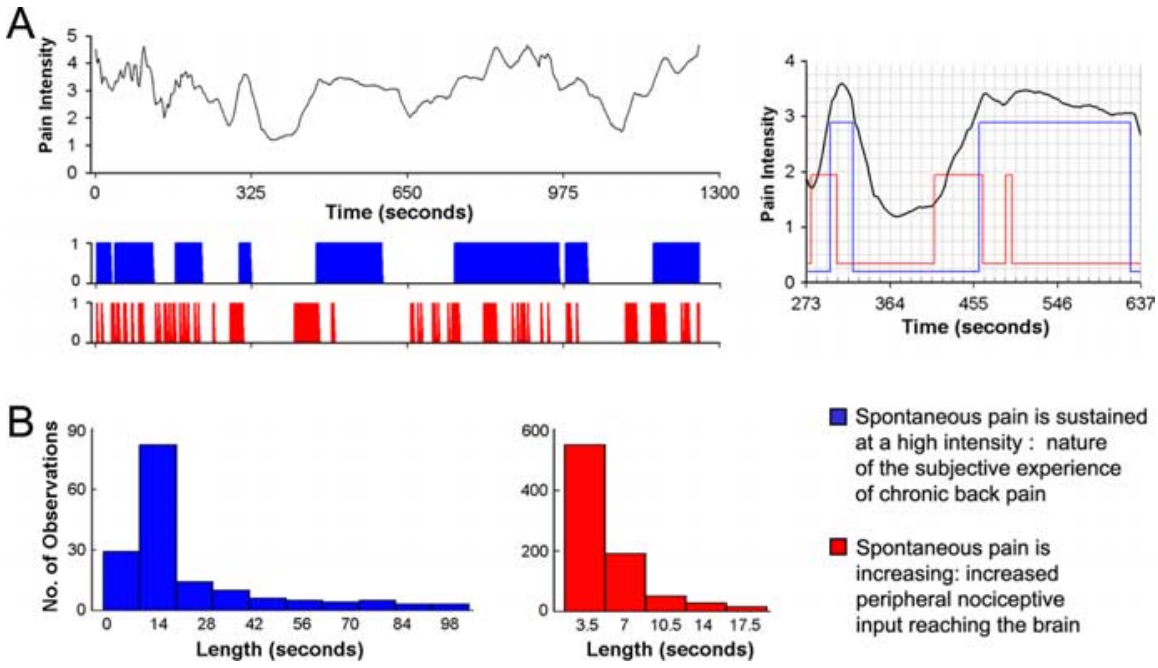

Figure 1. Ratings of fluctuations of spontaneous pain of CBP can be parceled into distinct phases, and each phase has a different time length distribution. $\boldsymbol{A}$, Top, Example rating of spontaneous fluctuations of pain in one (BP patient. The bottom panel shows two vectors derived from such ratings to search for brain activity BOLD signal. The first vector (blue) corresponds to time periods when the subjective rating (experience) of spontaneous pain intensity is high. It is the binarized high-low pain rating, relative to mean pain. The second vector (red) corresponds to time periods where ratings are rapidly increasing, which may correspond to increased nociceptive input to the brain. The right inset shows relationships between the three time-curves at a higher time resolution. $\boldsymbol{B}$, The length of the two vectors exhibit different distributions across all 13 CBP patients. The left panel is for time periods when spontaneous pain is high ( $n=161$ events; mean, $22.5 \mathrm{~s}$; mode, $10.5 \mathrm{~s} ; \mathrm{SD}, 22.1 \mathrm{~s}$ ), and the right panel is for time periods when spontaneous pain is increasing ( $n=866$ events; mean, $5.3 \mathrm{~s}$; mode, $3.5 \mathrm{~s} ; \mathrm{SD}, 3.1 \mathrm{~s}$ ).

Institute (MNI) space, http://www.bic.mni.mcgill.ca/cgi/icbm_view/]. This results in a $Z$-score map of statistically significant pain-related activity across different conditions. For random effects, cluster-based correction of the $Z$-statistic images was performed. The raw $Z$-statistic images from the group analysis were thresholded at $Z$-scores $>2.3$. For each resulting cluster of spatially connected voxels surviving the $Z$ threshold, a cluster probability threshold of $p=0.01$ was applied to the computed significance of that cluster, which corrects for multiple comparisons according to Gaussian random field theory (Friston et al., 1995).

Subtracting the visual rating task from the pain condition eliminated mainly posterior parietal cortex activity (attention-related activity). The group-averaged surrogate map was empty and subtracting it from the pain condition in study 1 had little effect on the overall activity map. Therefore, this subtraction was deemed not essential and was not included in study 2.

To determine behavioral parameters related to brain activity for spontaneous CBP, multiple pain-related parameters were covaried with high pain and with increasing pain conditions. Correlations were based on $z$ values obtained from the thresholded individual maps averaged for $1 \mathrm{cc}$ volume (27 voxels) around the peak coordinate of region of interest.

Average time course of BOLD response was calculated on an individual subject basis and then averaged across subjects. For each condition of interest, high spontaneous pain, or spontaneous pain increasing, preprocessed fMRI images are reaveraged relative to the start time of the condition, two brain volumes before the start time and four after start time, spanning in time from -7 to $+14 \mathrm{~s}$. Time courses for coordinates of interest are then extracted from each subject's brain, averaged over the 27-voxel neighborhood, and then averaged across subjects (see Figs. $2-4)$. Variability of these curves is expressed in SEM over the subject population.

Study 2. CBP patients from group 2 and healthy subjects from group 3 participated in this study. Functional MR data for both groups were acquired with a $3 \mathrm{~T}$ Siemens Trio whole-body scanner with EPI capability using the standard radio-frequency head coil. Multislice T2*-weighted echo-planar images were obtained with the following parameters: TR, $2.5 \mathrm{~s}$; TE, $70 \mathrm{~ms}$; flip angle, $90^{\circ}$; slice thickness, $3 \mathrm{~mm}$; in-plane resolution, $3.475 \times 3.475 \mathrm{~mm}^{2}$. The 36 slices covered the whole brain from the cerebellum to the vertex. An average of 400 volumes were acquired per event per condition in all participants. A T1-weighted anatomical MRI image was also acquired for each subject using the following parameters: TR, $2.1 \mathrm{~s}$; TE, 4.38 $\mathrm{ms}$; flip angle, $8^{\circ}$; FOV, $220 \mathrm{~mm}$; slice thickness, $1 \mathrm{~mm}$; in-plane resolution, $0.86 \times 0.86 \mathrm{~mm}^{2}$; number of sagittal slices, 160.

Similar to study 1 , image analysis to reveal significant brain activity based on changes in BOLD signal was performed on each subject's data using FMRIB FEAT (Jezzard et al., 2001) (www.fmrib.ox.ac.uk/fsl). The preprocessing of each subject's time-series of fMRI were identical to that used in study 1 . The fMRI signal was then linearly modeled on a voxel by voxel basis using FMRIB's FILM with local autocorrelation correction (Woolrich et al., 2001, 2004). One component was derived from each of spontaneous pain ratings (group 2), and the visual control ratings (groups 2 and 3). For thermal pain ratings, the high pain and increasing pain components were used to search for brain activity (groups 2 and 3). All four components were derived in a similar manner to that of study 1 (i.e., binarized relative to the mean). Each component (spontaneous pain, thermal pain, and visual) was considered to generate a hemodynamic response described by the convolution of the corresponding vector with a generalized hemodynamic response function (gamma function: lag, $6 \mathrm{~s}$; SD, 3 s). Head motion vector (derived from motion correction) was used at this level as a covariate of no interest. The significance of the model fit to each voxel time-series was calculated. Higherlevel analysis was done in a similar manner to study 1 , where average group activity maps were generated by subtracting visual maps from the thermal and spontaneous pain activity maps, using FEAT in a second level random effects group analysis. This results in a $Z$-score map of statistically significant pain-related activity across different pain conditions. A cluster-based correction of the $Z$-statistic images was performed thresholded at $Z$-scores $>2.3$ and a cluster probability threshold of $p=$ 0.01 was applied to correct for multiple spatial comparisons according to Gaussian random field theory (Friston et al., 1995).

Covariate analysis between brain activity and pain intensity was also performed for thermal and spontaneous pain where only mean pain intensity was examined. Correlations were based on $\mathrm{z}$ values obtained from the thresholded individual maps averaged for $1 \mathrm{cc}$ volume around the peak coordinate of region of interest.

\section{Results}

\section{Brain activity for spontaneous pain (study 1)}

We used ratings of fluctuations of pain, performed during fMRI scans, to derive two temporal components that may reflect different physiological states of chronic pain: (phase 1) time periods when spontaneous pain is sustained at a high intensity that may characterize the nature of the subjective experience of chronic pain, and (phase 2) time periods when spontaneous pain is transiently increasing, which may capture the peripheral nociceptive input reaching the brain (Fig. 1). Identification of brain activity for these two phases indicate that distinct nonoverlapping brain regions are involved in each phase, and that the temporal pattern of activity is also distinct for the two phases.

Brain activity for spontaneous fluctuations of CBP was determined in $13 \mathrm{CBP}$ patients (using a $1.5 \mathrm{~T}$ fMRI magnet). For time periods when spontaneous pain of CBP is high (sustained phase: binarized high, low relative to the mean of the ratings) (Fig. $1 \mathrm{~A}$ ), the group-averaged map contrasted with appropriate controls, with random-effects modeling showed activity in a single cluster within the medial prefrontal cortex (mPFC), in and around the 
rostral anterior cingulate (rACC) extending to the level of the genu (Fig. 2A). Two alternative routes have been proposed to mediate activity of $\mathrm{mPFC}$ in pain: (1) through forward projections from parietal, insular and cingulate regions encoding nociceptive information and (2) through midbrain and amygdala projections reflecting inputs from emotional and motivational circuitry (Price, 2000; Hunt and Mantyh, 2001; Apkarian et al., 2005). To distinguish between these, we performed the same contrast using fixedeffects model, which would reveal brain activity at a lower threshold level where the results can only be generalized to the subjects studied. The results showed additional bilateral activity in posterior thalamus, ventral striatum and extended amygdala, as well as larger activity spread within prefrontal cortex, and did not reveal any activity in parietal and insular regions (Fig. 2B), implying that when spontaneous pain of CBP is high and sustained it engages brain areas involved in emotion, cognition, and motivation (Davidson, 2002; Dolan, 2002; Phelps et al., 2004).

For the condition when spontaneous pain of CBP is increasing (transient phase: binarized times when spontaneous pain is increasing, which are times when pain ratings have a high and positive derivative) (Fig. $1 \mathrm{~A}$ ) contrasted with its controls with random-effects modeling showed activity in right anterior and posterior insula, secondary somatosensory cortex, multiple portions of mid cingulate (mACC), primary somatosensory region of the foot, and cerebellum (Fig. 2D). These activations correspond to the pattern observed classically for acute pain, encompassing regions subserving sensory and affective dimensions of pain (Talbot et al., 1991; Casey et al., 1996; Coghill et al., 1999; Price, 2000; Apkarian et al., 2005). We also investigated brain activity for the time periods when spontaneous pain of CBP is decreasing. The groupaverage contrasted with controls did not exhibit any significant increased or decreased brain activity.

Contrast and conjunction analyses indicated that brain activity for times when spontaneous pain is high and for times when spontaneous pain is increasing do not overlap. The temporal properties of the brain responses for the two phases of spontaneous pain were determined from the BOLD responses (Fig. 2C,E), which show profiles corresponding to the mode of the distribution of each phase (Fig. $1 B$ ), where the brain responses for high pain are longer lasting than the responses for increasing pain. Thus, the sustained and transient phases of spontaneous fluctuations of CBP seem to differentially activate either emotional regions of the brain or brain regions commonly observed for sensory processing of acute pain.
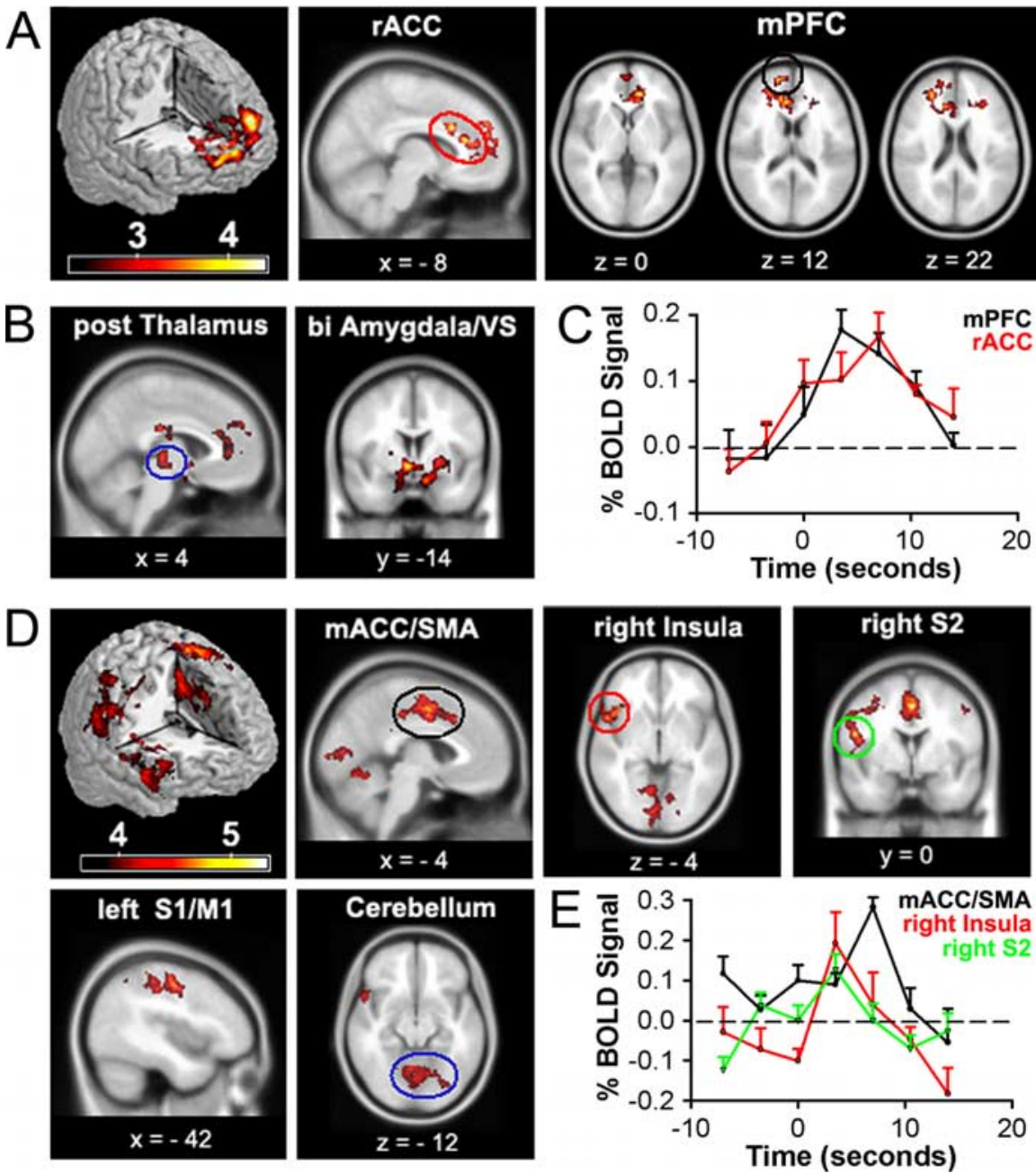

Figure 2. Brain activity for spontaneous CBP shows two distinct patterns: one for phases identified as high pain and another for increasing pain phases. $A$, Random-effects average brain activity in CBP patients for time periods in which spontaneous pain is thalamus, amygdala, and ventral striatum (VS). C, BOLD response for peak activations in $\operatorname{rACC}(10,22,28)$ and $\mathrm{mPFC}(18,60,12)$ for periods when spontaneous pain is high. Across-subject mean and SEMs are shown. $\boldsymbol{D}$, Random-effects analysis for periods spontaneous pain is increasing. Brain activity does not overlap with $A$ and is primarily located in right anterior insula, $\mathrm{mACC}$ and supplementary motor area (SMA), left primary somatosensory (S1), and motor (M1) regions, right secondary somatosensory cortex (S2), and cerebellum. Fixed-effect analysis did not reveal additional brain activity (data not shown). $\boldsymbol{E}$, BOLD response for pain is increasing. Across-subject mean and SEMs are shown. A complete list of activations is found in supplemental tables 2 and 3 , availableat www.jneurosci.org as supplemental material. Activity maps are presented in MNI space, $x, y$, and $z$ coordinates in millimeters.

Brain activity specific to intensity and duration of CBP (study 1 )

Given that the above brain activities were determined based on subjective ratings, it is imperative to establish their relationship to characteristics of CBP. Questionnaires and verbal ratings were used to determine duration and intensity of CBP pain, sensory and negative-affective dimensions of CBP, and levels of anxiety and depression. These characteristics were used in covariate analyses to determine their relationships to brain activity for CBP. A single cluster centered in the MPFC correlated with pain intensity for periods of high spontaneous CBP (Fig. 3A), with a peak correlation $R^{2}=0.81\left(p<10^{-5}\right)$. Another single cluster localized to right anterior insula correlated with duration of pain in years for periods of increases of spontaneous CBP (Fig. 3B), with a peak correlation $R^{2}=0.80\left(p<10^{-5}\right)$. Anxiety and depression indices showed no significant relationship with these conditions, and 

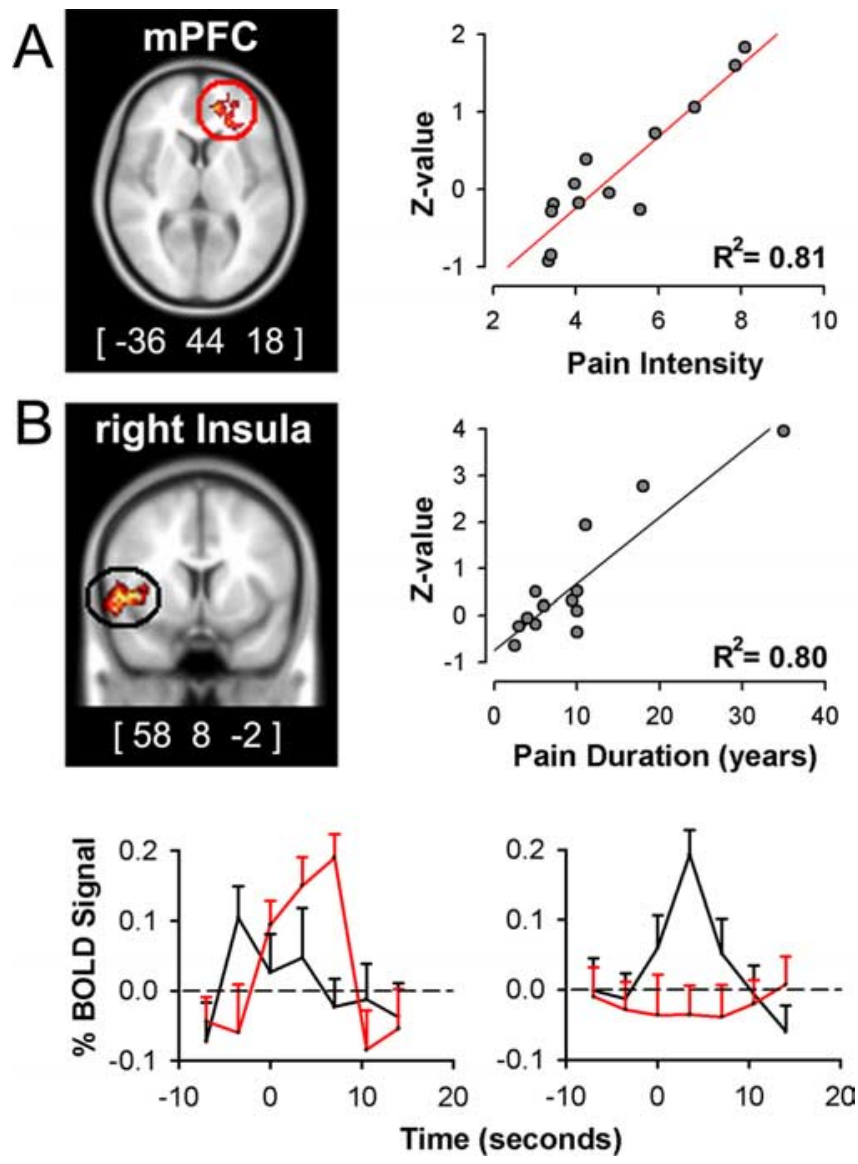

Figure 3. Intensity and duration of $C B P$ are correlated with distinct brain areas and for different phases of spontaneous fluctuations of CBP. $A$, Covariate analysis between pain intensity (mean rating of pain during scan) and brain activity for phases when spontaneous pain is high results in a single cluster in $\mathrm{mPFC}$, maximum across-subject correlation is at $(-36,44,18)$ $\left(R^{2}=0.81 ; p<0.01\right) . B$, Covariate analysis between pain duration (in years) and brain activity for phases when spontaneous pain is increasing results in right anterior insular cluster, with maximum correlation at $(58,8,-2)\left(R^{2}=0.80 ; p<0.01\right)$. C, Time courses for peak activations in $\mathrm{mPFC}$ (red) and insula (black) for periods when spontaneous pain is high (left) and when spontaneous pain is increasing (right). Across-subject mean and SEMs are shown. Activity maps are presented in MNI space, $x, y$, and $z$ coordinates in millimeters.

did not influence pain intensity and duration correlations when used as covariates of no interest (to correct for their contribution to total variance). The temporal properties for the activity in these two areas (Fig. 3C) indicate that (1) the insular region correlated with pain duration is active during increases in pain whereas $\mathrm{mPFC}$ region is not, and (2) the $\mathrm{mPFC}$ region best correlated to intensity of pain is active during high pain (for a longer duration than the insula) and in this time period the insular activity is transient and precedes the $\mathrm{MPFC}$ activity. This analysis indicates that intensity and duration of CBP can be explained by the differential activity for the two phases of spontaneous pain.

\section{Relating brain atrophy and activity (study 1)}

Multiple studies indicate that activity in $\mathrm{MPFC}$ and dorsolateral prefrontal cortex (DLPFC) are inversely related (Mayberg et al., 1999; Northoff et al., 2000). We demonstrated previously that DLPFC gray matter density is decreased in CBP (Apkarian et al., 2004b). To identify the relationship between brain activity during spontaneous pain and DLPFC activity, specifically for the region that shows maximal atrophy (DLPFCa), we determined correla- tions between DLPFCa, mPFC, and insula for the two phases of spontaneous pain.

Left and right DLPFCa show a negative correlation with activity in $\mathrm{mPFC}(p<0.001)$ (Fig. $4 A)$, but not with insula $(p>0.7)$, for times when spontaneous pain of CBP is high. The DLPFCa signals are active before the increase in $\mathrm{MPFC}$ activity, and switch to deactivation for the time period where $\mathrm{mPFC}$ signal is sustained (Fig. $4 B$ ). Time course analysis of DLPFCa for times when spontaneous pain is transiently increasing show, similarly to $\mathrm{mPFC}$, no overall signal change. However, correlations between mPFC, DLPFCa, and anterior insula are all highly significantly positive $\left(R^{2}\right.$ ranging from 0.64 to $0.68 ; p<0.01$ for each case) (data not shown).

The shift in correlations coupled with the observed activity implies a minimal dynamical model for nociceptive information processing in CBP. The increasing phase of spontaneous pain is most likely caused by afferent nociceptive input accessing the cortex because it activates the acute pain sensory system and coactivates $\mathrm{mPFC}$ and DLPFCa. When this afferent barrage results in sustained $\mathrm{mPFC}$ activity, perception of ongoing pain is increased, and $\mathrm{mPFC}$ and DLPFCa inhibit each other. The competition between MPFC and DLPFCa, perhaps coupled with enhanced spinal-prefrontal projections, determine the duration of sustained mPFC activity and, thus, also for heightened ongoing pain by increasing emotional salience. Note that neither this model nor the above analysis is based on alterations in the $\mathrm{mPFC}$ and DLPFC activity relationship.

\section{Brain activity for thermal pain is distinct from activity for sustained spontaneous CBP (study 2)}

In this study, we use fMRI to explore both common and unique brain circuitry underlying CBP and acute pain. We determined brain responses (using a 3.0 T fMRI magnet) to thermal stimuli applied to the back in 11 CBP patients and in 11 age- and sexmatched normal subjects and, in this new CBP patient group, we again determined responses to ratings of spontaneous fluctuations of CBP. The experiment was performed to (1) replicate the findings of brain activity for spontaneous pain in CBP in a second group, (2) to directly differentiate between brain activity for acute thermal pain and spontaneous pain of CBP, and (3) to test that our method of using continuous ratings of perceived pain results in brain activity corresponding to earlier studies of acute thermal pain in normal subjects (where subjects usually passively attend noxious stimuli) (Talbot et al., 1991; Casey et al., 1996; Coghill et al., 1999; Apkarian et al., 2005). Normal healthy subjects' and CBP patients' brain activity to thermal painful stimuli were studied using the same approach as delineated for spontaneous pain of CBP. Thermal stimulus characteristics were chosen to approximate the properties of fluctuations of spontaneous pain in CBP, both in intensity and range of stimulus durations. There were no differences between CBP patients and normal controls regarding the intensity and temporal profile of thermal pain ratings (Fig. 5A). Stimulus ratings in both groups were used to generate a new map now in response to noxious heat for time periods when pain ratings were high and for increasing, as well as for spontaneous pain in CBP. Resultant brain activities are subtracted from appropriate controls, and random-effects model used to identify brain regions involved in each condition. Brain activity in CBP for high pain of spontaneous fluctuations of CBP resulted mainly in $\mathrm{mPFC}$ activity, including the anterior cingulate at the genu, and an additional cluster of activity in the posterior parietal cortex. This, result then replicates the main observation of study 1 in a new population of CBP patients, on a different 
(higher strength) magnet. Responses to thermal stimuli for periods when pain is high were similar between controls and CBP (across group $t$ test shows no brain activity differences), and activated mainly bilateral insula, secondary somatosensory cortex, cingulate cortex, and right DLPFC (Fig. 5B), in agreement with earlier observations (Price, 2000; Derbyshire et al., 2002; Apkarian et al., 2005). In CBP patients, conjunction analysis indicated no overlap between activity for sustained spontaneous pain and thermal pain. Brain activity for increases in thermal pain (supplemental Fig. 2, available at www. jneurosci.org as supplemental material) was also similar to brain activity for the periods when thermal pain was high, and the contrast between the groups again showed no difference.

We again performed a covariate analysis between pain intensity and brain activity, now for all three conditions: (1) spontaneous pain of CBP, (2) thermal pain in CBP, and (3) thermal pain in normal controls. Sustained spontaneous CBP was again correlated only with $\mathrm{MPFC}$ activity (replicating the result of study 1), whereas thermal pain intensity was best correlated with insular activity in both CBP and control subjects. The reverse was also true: $\mathrm{mPFC}$ activity was not correlated with pain intensity for thermal pain in CBP and control subjects, and insular activity was not correlated with sustained spontaneous CBP (Fig. 6). Therefore, continuous pain ratings give rise to results that closely approximate earlier findings for brain regions activated in acute pain and for brain regions coding stimulus intensity for acute pain (Derbyshire et al., 1997; Coghill et al., 1999; Price, 2000; Buchel et al., 2002; Apkarian et al., 2005). We also demonstrate a double-dissociation between sensory and emotional regions of the brain in encoding pain intensity for acute pain in contrast to sustained spontaneous pain, with sensory regions (especially insula) coding the perceived magnitude of thermal pain in both normal subjects and CBP patients, and the emotional region ( $\mathrm{mPFC}$ ) coding the magnitude of spontaneous pain of CBP.

\section{Discussion}

The results provide new insights regarding neural mechanisms of spontaneous pain in CBP and confirm our primary hypotheses. We observe that spontaneous pain in CBP transiently engages anterior insula and other sensory-related regions seen in acute pain, when spontaneous pain is increasing. This is replaced by sustained mPFC activity as long as the pain remains high. Whereas insular activity reflects chronicity of CBP, mPFC activity reflects intensity of $\mathrm{CBP}$ and is likely to be enhanced by atrophy in DLPFC. Moreover, we show that brain activity for sustained spontaneous CBP is distinct from thermal pain even within the same subjects, implying that the sensory, emotional, and cognitive properties of spontaneous pain of CBP are very different from acute pain. Whether these differences are attributable to the chronicity of the condition or to the spontaneous pain per se remains to be explored.

Parts of mPFC are activated during anticipation of pain (Porro et al., 2002), anticipation of placebo (Wager et al., 2004), and when unpleasantness of acute pain is enhanced (Lorenz et al., 2002). However, the present study is unique in showing that
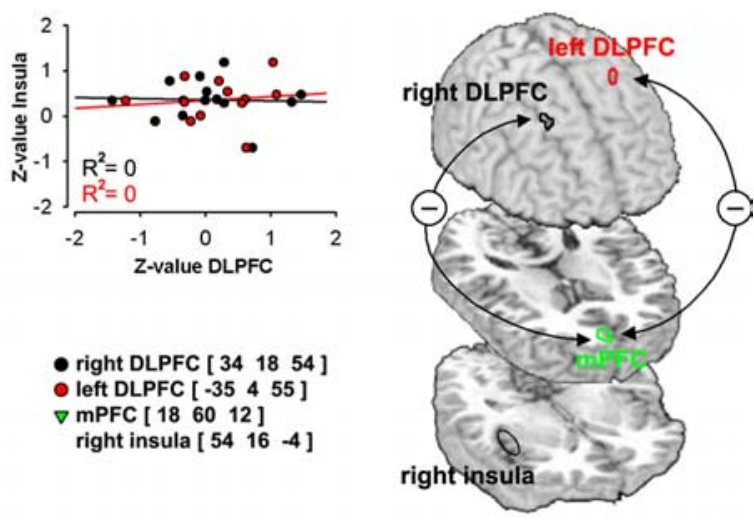

Figure 4. Activity in right and left DLPFC locations with peak atrophy in CBP (Apkarian et al., 2004b), during high pain, show an inverse relationship between $\mathrm{mPFC}$ but not with insula. $\boldsymbol{A}$, Across-subject correlations between brain activity for right and left correlations are depicted on the brain. Note that these relations are only true during high pain phase. All four areas are significantly correlations are depicted on the brain. Note that these relations are only true during
positively correlated with each other during increasing phase of spontaneous CBP.

some pain conditions (sustained high spontaneous CBP) map only to mPFC activity. Multiple lines of evidence implicate paracingulate the mPFC (mPFC and rACC at the genu) in emotions (Dolan, 2002; Phelps et al., 2004), and response conflict and detection of unfavorable outcomes (Ridderinkhof et al., 2004). It is involved in persistence of emotion after the offset of an elicitor (elicitor in our case is the nociceptive input), and modulates the time course of emotional response, particularly recovery time (Davidson, 2002). In addition, rACC at the genu seems involved when subjects reflect on emotions that they are currently experiencing (Gusnard et al., 2001), and also in "theory of mind" tasks (Gallagher and Frith, 2003). Therefore, sustained activity in the region during spontaneous CBP most likely reflects a negative emotional state in reference to the self. Multiple factors can contribute to the sustained mPFC activity after decrement of the nociceptive elicitor: (1) the nature of the pain (more unpleasant than acute pain); (2) enhanced spinal prefrontal projections through pathways outside the spinothalamic tract, such as spinoparabrachial, spinostriatal, and spinoreticular pathways (Willis and Westlund, 1997; Gauriau and Bernard, 2002; Braz et al., 2005), as predicted in animal models of neuropathic pain (Hunt and Mantyh, 2001); (3) the interaction between mPFC and DLPFCa. The DLPFC is involved in working memory (Cohen et al., 1997; Courtney et al., 1997); its activity is usually inversely related with mPFC activity (Mayberg et al., 1999; Northoff et al., 2000), as shown for pain as well (Lorenz et al., 2002). Emotional reasoning enhances activation in $\mathrm{MPFC}$ and suppresses DLPFC activity, and this pattern is reversed for emotionally neutral reasoning (Goel and Dolan, 2003; Levesque et al., 2003). Because DLPFC atrophy in CBP is related to duration and intensity of CBP (Apkarian et al., 2004b), the demonstrated relationship between DLPFCa and mPFC may provide a link between brain atrophy and sustained suffering of back pain.

The increasing phase of spontaneous CBP is characterized by transient activity in areas commonly observed for acute pain. Therefore, the activity pattern during increases in spontaneous pain is most likely a reflection of the properties of the nociceptive elicitor (afferent input). Of these regions, the anterior insula accounted for $70-80 \%$ of the variance of duration of CBP. Studies show that the anterior insula is activated for pain in contrast to 

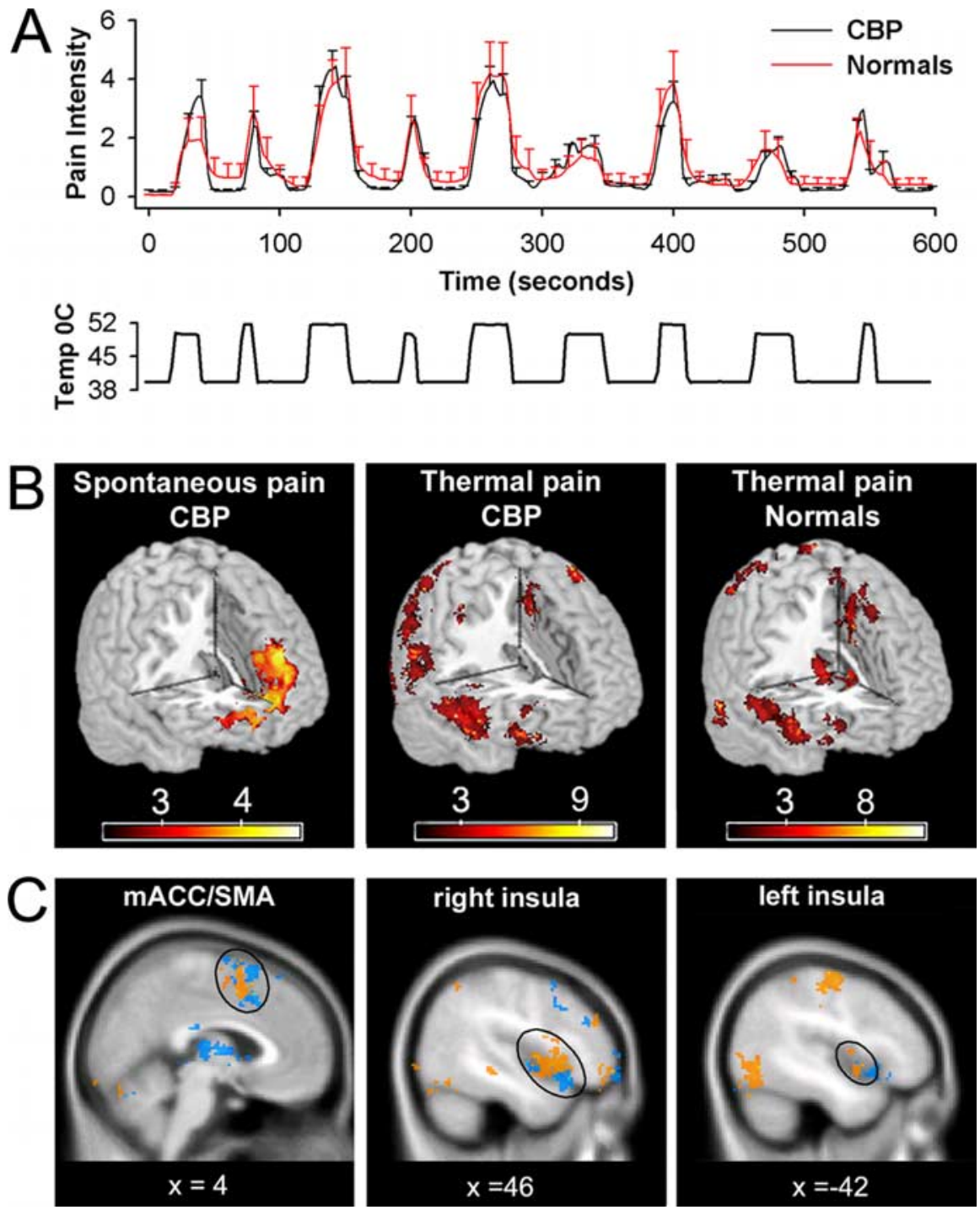

Figure 5. Brain activity for spontaneous pain of CBP does not show any overlap with that of thermal pain in CBP and healthy normal subjects. $\boldsymbol{A}$, Average pain ratings for thermal stimulus applied to the back in CBP patients $(n=11)$ and matched normal controls $(n=11)$. There is no significant difference in pain ratings between the two groups. Bottom shows temperature profile of the stimulus applied to the back. $\boldsymbol{B}$, Random-effects average brain activity for high pain of spontaneous CBP includes mPFC and rostral parts of the anterior cingulate. It is similar to the brain activity pattern observed for spontaneous pain in study 1 (compare Fig. $1 \mathrm{~A}$ ) and does not encompass brain areas activated for noxious heat in CBP (middle) and matched healthy controls (right). $C$, Similar brain activity patterns are seen for noxious heat applied to the back in (BP (orange) and in healthy normal subjects (blue). It includes the bilateral insular cortex, medial ACC, and supplementary motor areas, in addition to cerebellum and somatosensory regions. Activity maps are presented in MNI space, $x, y$, and $z$ coordinates in millimeters. Detailed activity maps for $\boldsymbol{B}$ and complete lists of activated areas are found in supplemental Figure 1 and Table 4, available at www. jneurosci.org as supplemental material.

nonpainful touch (Price, 2000; Apkarian et al., 2005) in anticipation of pain (Ploghaus et al., 1999), pain empathy (Singer et al., 2004); insular lesions can lead to pain neglect-like behavior (Berthier et al., 1988), and stimulation within insula evokes painful experiences (Ostrowsky et al., 2002). Therefore, transient insular activity during spontaneous CBP provides a sensory-related nociceptive elicitor signal, which is amplified the longer a subject suffers from CBP. We also demonstrate a shift in the insula responses between thermal stimuli and spontaneous pain, for the painful stimulus insula shows sustained activity that reflects perceived intensity whereas during spontaneous pain it is only transiently active and does not reflect stimulus intensity. Therefore, the spatiotemporal properties of acute pain and spontaneous
CBP are clearly distinct, and the activity in the latter more likely are sustained through nociceptive pathways outside of the spinothalamic tract.

\section{What is chronic back pain?}

The current study is the latest of a series we have been conducting to examine the brain in CBP. Together, how do these studies impact the current viewpoint regarding CBP? The common clinical approach to CBP is to relate its behavioral manifestations to the site of injury. Although some CBP patients have identifiable structural or mechanical cause for their pain, most do not (Cavanaugh and Weinstein, 1994; Boos et al., 1995; Deyo, 1998). Given the poor association between structural abnormalities to pain, other nonspecific variables have been proposed as predictors of clinical outcome, like demographics including age, gender, and education (Boos et al., 1995), psychosocial factors such as level of depression, anxiety, pain catastrophizing, fear and/or helplessness, job satisfaction, and environmental reinforcers such as compensation and litigation (Greenough, 1993; Keefe et al., 2004). Despite this long list, incorporating these parameters accounts for a relatively small portion of the variance of CBP (e.g., a previous such report can explain only $25 \%$ of the variance of intensity of back pain) (Peters et al., 2005).

In contrast, examining CBP from the viewpoint of the brain indicates that $\mathrm{CBP}$, regardless of whether it is secondary to fracture, inflammatory joint disease, postsurgical, combinations of these, or idiopathic (Deyo and Weinstein, 2001), presents a well defined set of abnormalities. It is associated with a specific pattern of brain chemical changes (Grachev et al., 2000) that are consistent with decreased gray matter density in DLPFC (Apkarian et al., 2004b), which in turn may account for heightened mPFC activity and decreased ability in emotional-decision making (Apkarian et al., 2004a). These brain parameters account for over $70-80 \%$ of the variance for intensity and duration of CBP. Therefore, they must be considered an integral part of the clinical state of CBP. The present results show that spontaneous CBP engages the emotional-mentalizing region of the brain into a state of continued negative emotions (suffering) regarding the self, punctuated by occasional nociceptive inputs that perpetuate the state. In 1968 it was postulated that "the frontal cortex may play a particularly significant role in mediating between cognitive activities and motivational-affective features of pain" (Melzack and Casey, 1968). The brain activity we show here for CBP identifies this prefrontal circuitry. The sustained activity of which must be related to the maladaptive psychological and behavioral cost asso- 
[ $\left.\begin{array}{lll}42 & 2 & -2\end{array}\right]$
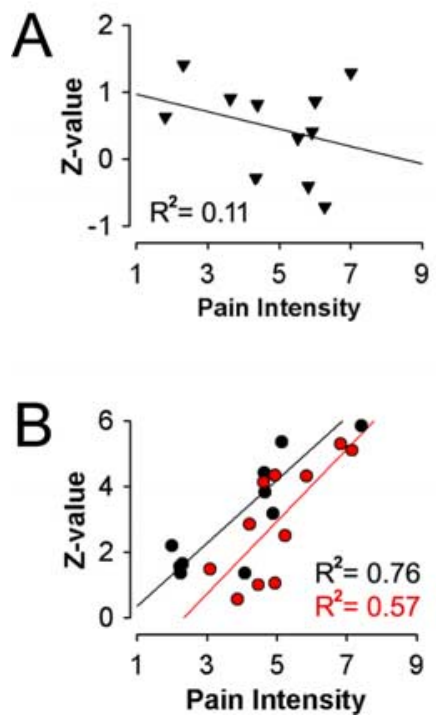

right Insula
MPFC $\left[\begin{array}{lll}-18 & 64 & 16\end{array}\right]$
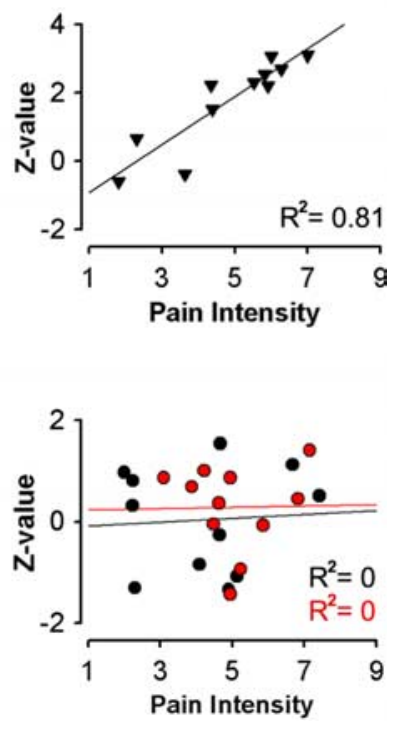

Figure 6. Pain intensity for spontaneous CBP and thermal pain are encoded in different brain regions. $A$, Pain intensity for spontaneous pain of $\mathrm{CBP}$ (mean rating of pain during scan) exhibits a significant positive correlation with $\mathrm{MPFC}$ activity $(p<0.001$, replicating the result shown in Fig. $3 A$ ) and is uncorrelated with insular activity. $\boldsymbol{B}$, Pain intensity for thermal pain in both (BP patients and normal subjects is best correlated with insular activity $(p<0.01)$ and shows no correlation with $\mathrm{mPFC}$ activity $(x, y$, and $z$ coordinates in millimeters).

ciated with chronic pain (Melzack and Casey, 1968; Price, 2000; Dworkin, 2002).

\section{References}

Apkarian AV, Bushnell MC, Treede RD, Zubieta JK (2005) Human brain mechanisms of pain perception and regulation in health and disease. Eur J Pain 9:463-484.

Atkinson JH (2004) Chronic back pain: searching for causes and cures. J Rheumatol 31:2323-2325.

Berthier M, Starkstein S, Leiguarda R (1988) Asymbolia for pain: a sensorylimbic disconnection syndrome. Ann Neurol 24:41-49.

Birklein F, Riedl B, Sieweke N, Weber M, Neundorfer B (2000) Neurological findings in complex regional pain syndromes-analysis of 145 cases. Acta Neurol Scand 101:262-269.

Boos N, Rieder R, Schade V, Spratt KF, Semmer N, Aebi M (1995) 1995 Volvo Award in clinical sciences. The diagnostic accuracy of magnetic resonance imaging, work perception, and psychosocial factors in identifying symptomatic disc herniations. Spine 20:2613-2625.

Braz JM, Nassar MA, Wood JN, Basbaum AI (2005) Parallel "pain" pathways arise from subpopulations of primary afferent nociceptor. Neuron 47:787-793.

Buchel C, Bornhovd K, Quante M, Glauche V, Bromm B, Weiller C (2002) Dissociable neural responses related to pain intensity, stimulus intensity, and stimulus awareness within the anterior cingulate cortex: a parametric single-trial laser functional magnetic resonance imaging study. J Neurosci 22:970-976.

Casey KL, Minoshima S, Morrow TJ, Koeppe RA (1996) Comparison of human cerebral activation pattern during cutaneous warmth, heat pain, and deep cold pain. J Neurophysiol 76:571-581.

Cavanaugh JM, Weinstein JN (1994) Low back pain: epidemiology, anatomy and neurophysiology. In: Textbook of pain (Wall PD, Melzack R, eds), pp 441-456. Edinburgh: Churchill Livingstone.

Clauw DJ, Williams D, Lauerman W, Dahlman M, Aslami A, Nachemson AL, Kobrine AI, Wiesel SW (1999) Pain sensitivity as a correlate of clinical status in individuals with chronic low back pain. Spine 24:2035-2041.

Coghill RC, Sang CN, Maisog JM, Iadarola MJ (1999) Pain intensity processing within the human brain: a bilateral, distributed mechanism. J Neurophysiol 82:1934-1943.

Cohen JD, Perlstein WM, Braver TS, Nystrom LE, Noll DC, Jonides J, Smith
EE (1997) Temporal dynamics of brain activation during a working memory task. Nature 386:604-608.

Courtney SM, Ungerleider LG, Keil K, Haxby JV (1997) Transient and sustained activity in a distributed neural system for human working memory. Nature 386:608-611.

Davidson RJ (2002) Anxiety and affective style: role of prefrontal cortex and amygdala. Biol Psychiatry 51:68-80.

Derbyshire SW, Jones AK, Gyulai F, Clark S, Townsend D, Firestone LL (1997) Pain processing during three levels of noxious stimulation produces differential patterns of central activity. Pain 73:431-445.

Derbyshire SW, Jones AK, Creed F, Starz T, Meltzer CC, Townsend DW, Peterson AM, Firestone L (2002) Cerebral responses to noxious thermal stimulation in chronic low back pain patients and normal controls. NeuroImage 16:158-168.

Deyo RA (1998) Low-back pain. Sci Am 279:48-53.

Deyo RA, Weinstein JN (2001) Low back pain. N Engl J Med 344:363-370.

Dolan RJ (2002) Emotion, cognition, and behavior. Science 298:1191-1194.

Dworkin RH (2002) An overview of neuropathic pain: syndromes, symptoms, signs, and several mechanisms. Clin J Pain 18:343-349.

Foss JM, Apkarian AV, Chialvo DR (2006) Dynamics of pain: fractal dimension of temporal variability of spontaneous pain differentiates between pain states. J Neurophysiol 95:730-736.

Friston KJ, Holmes AP, Worsley KJ, Poline J-B, Frith CD, Frackowiak RS (1995) Statistic parametric maps in functional imaging: a general linear approach. Hum Brain Mapp 2:189-210.

Gallagher HL, Frith CD (2003) Functional imaging of "theory of mind." Trends Cogn Sci 7:77-83.

Gauriau C, Bernard JF (2002) Pain pathways and parabrachial circuits in the rat. Exp Physiol 87:251-258.

Giesecke T, Gracely RH, Grant MA, Nachemson A, Petzke F, Williams DA, Clauw DJ (2004) Evidence of augmented central pain processing in idiopathic chronic low back pain. Arthritis Rheum 50:613-623.

Goel V, Dolan RJ (2003) Reciprocal neural response within lateral and ventral medial prefrontal cortex during hot and cold reasoning. NeuroImage 20:2314-2321.

Grachev ID, Fredrickson BE, Apkarian AV (2000) Abnormal brain chemistry in chronic back pain: an in vivo proton magnetic resonance spectroscopy study. Pain 89:7-18.

Greenough CG (1993) Results of treatment of lumbar spine disorders. Effects of assessment techniques and confounding factors. Acta Orthop Scand Suppl 251:126-129.

Gusnard DA, Akbudak E, Shulman GL, Raichle ME (2001) Medial prefrontal cortex and self-referential mental activity: relation to a default mode of brain function. Proc Natl Acad Sci USA 98:4259-4264.

Harstall C, Ospina M (2003) How prevalent is chronic pain? Pain Clinical Updates 11:1-4.

Hunt SP, Mantyh PW (2001) The molecular dynamics of pain control. Nat Rev Neurosci 2:83-91.

Jezzard P, Mathews P, Smith SM (2001) Functional MRI: an introduction to methods. Oxford, UK: Oxford UP.

Keefe FJ, Rumble ME, Scipio CD, Giordano LA, Perri LM (2004) Psychological aspects of persistent pain: current state of the science. J Pain 5:195-211.

Levesque J, Eugene F, Joanette Y, Paquette V, Mensour B, Beaudoin G, Leroux JM, Bourgouin P, Beauregard M (2003) Neural circuitry underlying voluntary suppression of sadness. Biol Psychiatry 53:502-510.

Lorenz J, Cross DJ, Minoshima S, Morrow TJ, Paulson PE, Casey KL (2002) A unique representation of heat allodynia in the human brain. Neuron 35:383-393.

Mayberg HS, Liotti M, Brannan SK, McGinnis S, Mahurin RK, Jerabek PA, Silva JA, Tekell JL, Martin CC, Lancaster JL, Fox PT (1999) Reciprocal limbic-cortical function and negative mood: converging PET findings in depression and normal sadness. Am J Psychiatry 156:675-682.

Melzack R, Casey K (1968) Sensory, motivational, and central control determinants of pain. In: The skin senses (Kenshalo DR, ed), pp 423-443. Springfield: Charles C. Thomas.

Merskey H, Bogduk N (1994) Descriptions of chronic pain syndromes and definitions of pain terms, Ed 2. Seattle: International Association for the Study of Pain.

Northoff G, Richter A, Gessner M, Schlagenhauf F, Fell J, Baumgart F, Kaulisch T, Kotter R, Stephan KE, Leschinger A, Hagner T, Bargel B, Witzel T, Hinrichs H, Bogerts B, Scheich H, Heinze HJ (2000) Func- 
tional dissociation between medial and lateral prefrontal cortical spatiotemporal activation in negative and positive emotions: a combined fMRI/ MEG study. Cereb Cortex 10:93-107.

Ostrowsky K, Magnin M, Ryvlin P, Isnard J, Guenot M, Mauguiere F (2002) Representation of pain and somatic sensation in the human insula: a study of responses to direct electrical cortical stimulation. Cereb Cortex $12: 376-385$.

Peters ML, Vlaeyen JW, Weber WE (2005) The joint contribution of physical pathology, pain-related fear and catastrophizing to chronic back pain disability. Pain 113:45-50.

Phelps EA, Delgado MR, Nearing KI, LeDoux JE (2004) Extinction learning in humans: role of the amygdala and vmPFC. Neuron 43:897-905.

Ploghaus A, Tracey I, Gati JS, Clare S, Menon RS, Matthews PM, Rawlins JN (1999) Dissociating pain from its anticipation in the human brain. Science 284:1979-1981.

Porro CA, Baraldi P, Pagnoni G, Serafini M, Facchin P, Maieron M, Nichelli P (2002) Does anticipation of pain affect cortical nociceptive systems? J Neurosci 22:3206-3214.

Price DD (2000) Psychological and neural mechanisms of the affective dimension of pain. Science 288:1769-1772.

Ridderinkhof KR, Ullsperger M, Crone EA, Nieuwenhuis S (2004) The role of the medial frontal cortex in cognitive control. Science 306:443-447.

Sah DW, Ossipo MH, Porreca F (2003) Neurotrophic factors as novel therapeutics for neuropathic pain. Nat Rev Drug Discov 2:460-472.
Sindrup SH, Andersen G, Madsen C, Smith T, Brosen K, Jensen TS (1999) Tramadol relieves pain and allodynia in polyneuropathy: a randomised, double-blind, controlled trial. Pain 83:85-90.

Singer T, Seymour B, O’Doherty J, Kaube H, Dolan RJ, Frith CD (2004) Empathy for pain involves the affective but not sensory components of pain. Science 303:1157-1162.

Talbot JD, Marrett S, Evans AC, Meyer E, Bushnell MC, Duncan GH (1991) Multiple representations of pain in human cerebral cortex. Science 251:1355-1358.

Tasker RR, de Carvallo G, Dostrovsky JO (1991) The history of central pain syndromes, with observations concerning pathophysiology and treatment. In: Pain and central nervous system disease: the central pain syndrome (Casey KL, ed), pp 31-58. New York: Raven.

Wager TD, Rilling JK, Smith EE, Sokolik A, Casey KL, Davidson RJ, Kosslyn SM, Rose RM, Cohen JD (2004) Placebo-induced changes in FMRI in the anticipation and experience of pain. Science 303:1162-1167.

Willis WD, Westlund KN (1997) Neuroanatomy of the pain system and of the pathways that modulate pain. J Clin Neurophysiol 14:2-31.

Woolrich MW, Ripley BD, Brady M, Smith SM (2001) Temporal autocorrelation in univariate linear modeling of FMRI data. NeuroImage 14:1370-1386.

Woolrich MW, Behrens TE, Beckmann CF, Jenkinson M, Smith SM (2004) Multilevel linear modelling for FMRI group analysis using Bayesian inference. NeuroImage 21:1732-1747. 